\title{
Application of Python language in UOE molding simulation of pipeline steel
}

\author{
Lian Yeda ${ }^{1}$, Zhang Bing ${ }^{1, a}$ and Wu Renqiang ${ }^{1}$ \\ ${ }^{1}$ Northwestern Polytechnical University, School of Mechanics, Civil Engineering and Architecture, Xi'an, 710129,China
}

\begin{abstract}
The ABAQUS plug-in interface based on Python language realizes geometrical design and automatic modeling of gas pipeline UOE molding, which solves the cumbersome problem of manually building complex geometric models. In this study, the algorithm for different sizes of pipelines corresponding to different molds was designed. At the same time, as the ABAQUS kernel scripting program was written, a GUI interface was developed. The interface was used to realize automatic modeling and analysis and control of the calculation work, which laid a solid foundation for practical engineering application analysis.
\end{abstract}

\section{Introduction}

\subsection{Introduction to Python language}

Python is an object-oriented interpreted computer programming language. It was invented by the Dutch Guido van Rossum in 1989. In 1991, he released the first public version of Python. The basic code base provided by the Python language is very rich, covering the advantages of other programming languages at that time. Since its official release, the development history has been more than twenty years, and it has developed to a relatively stable stage. Teng Jun et al. [1] conducted secondary development based on ABAQUS using Python. The interface of Python language exists in finite element analysis software ABAQUS, therefore, through the authoring of the Python language script code and the ABAQUS secondary development interface [2-3], the parameterized automatic modeling process can be realized by combining the GUI interface created by the plug-in toolkit in ABAQUS with the Python language script [4].

\subsection{Introduction to UOE molding}

The production process of pipeline steel is of great significance for the safe transportation of oil and natural gas. Every country in the world pays great attention to the transportation of oil and natural gas, because the pipelines cross over a long distance and pass through land and sea. Once there is a problem in the transportation process, it will threaten the safety of people and property. UOE is a pipe forming method and is mainly divided into $U$ molding, $\mathrm{O}$ molding, and expanding. Figure 1 and Figure 2 show the actual pictures of pipeline steel forming. The efficiency of forming welded pipes is very high and it is widely used in various countries in the world.

\footnotetext{
${ }^{a}$ Corresponding author: 970427933@qq.com
} 


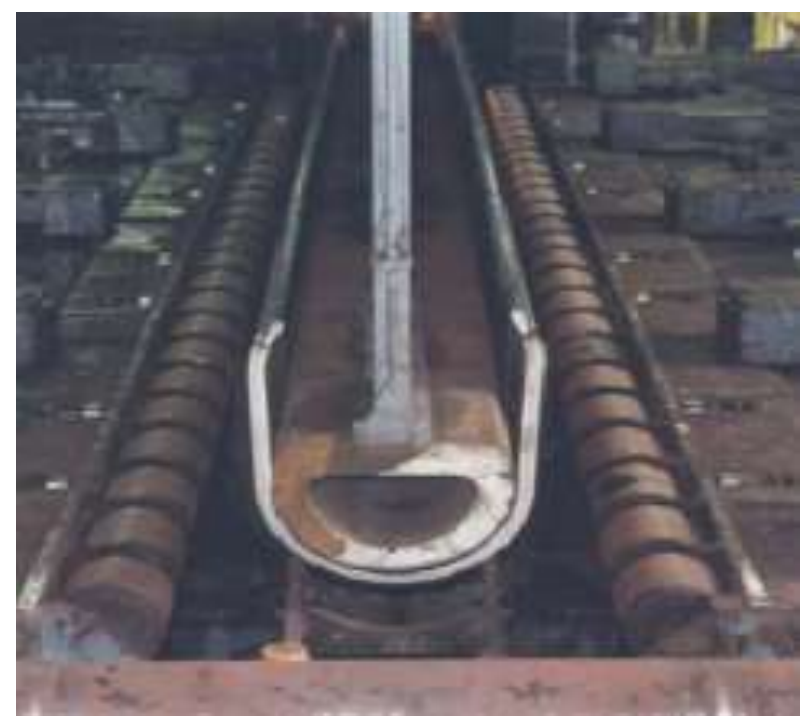

Figure 1. Pipeline steel pipe in $U$ molding process.

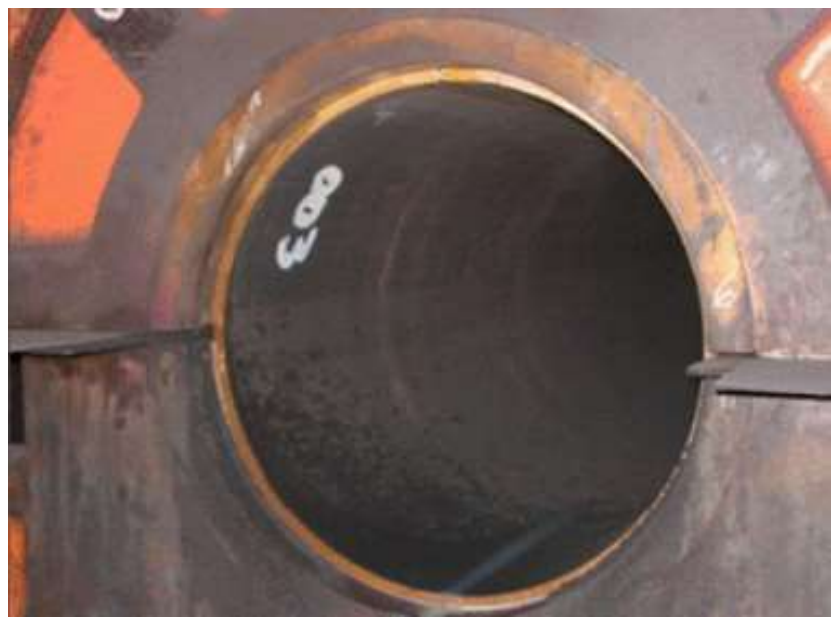

Figure 2. Pipeline steel pipe in $\mathrm{O}$ molding process.

\section{The development of visual interface}

\subsection{Introduction to GUI functions in ABAQUS}

The finite element software ABAQUS provides some interfaces for secondary development. Therefore, it is possible to use a computing platform to compile a simple modeling program and achieve rapid modeling. The secondary development [5-6] mainly has the following ways:

(1) Some new models can be easily defined by user subroutines to suit their own needs and achieve the purpose of controlling the calculation process and results.

(2) By changing the initial environment, more work can be saved.
(3) The establishment of finite element models and computational analysis of models can be achieved by compiling kernel scripts.

(4) Create a new visual user interface through the compilation of Python language.

\subsection{Parameterized design for pipe forming of pipeline steel}

It takes about 3 hours to build a simulation using ABAQUS's own graphical interface. However, it has different die sizes for different welded pipe sizes, which greatly increases the modeling time and the workload during simulation. For such a problem, it can be solved by establishing a parametric model. Firstly, the basic parameters of the initial sheet must be determined. As shown in Fig. 3, the relevant parameters of the sheet material are width (W), length (L), thickness $(\mathrm{T})$ and sheet yield strength (q). Based on the parameters of the sheet material, the relevant parameters of the mold can be determined by using the function relationship. Secondly, the positions of the molds and the panels in the molding process are determined. Finally, these basic parameters can be input through a graphical interface for modeling. The establishment of the sheet metal model and the definition of the material are completed, and the corresponding tube molds are also modeled. The entire complex modeling work is completely established by the visualized graphical parameter modeling program. The entire modeling process only takes about 2 minutes.

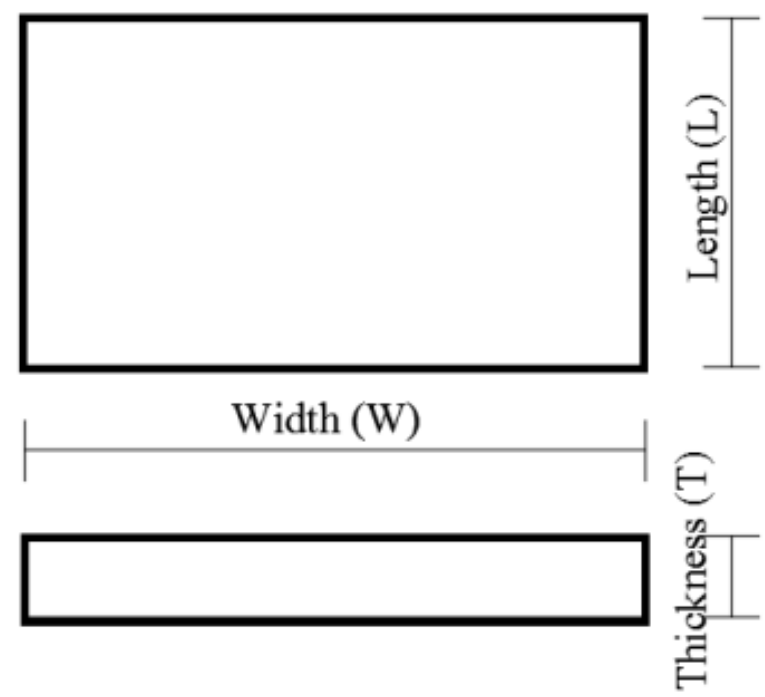

Figure 3. Basic dimensions of sheet. 


\subsection{The Design of graphical visual interface}

Based on the parameters determined in the parametric design of the simulation, this article establishes a visual graphical interface input by simplifying the complex parameters of the model. The process of writing graphical interfaces directly through code functions is pretty complicated. In this process, it not only needs a certain programming basis, but also needs to be very familiar with the functions and application methods of objects. In order to solve this kind of problem, this article chooses a relatively simple and effective method, which is to use the package (Really Simple GUI), which already provides some edited basic controls. Users can create components more conveniently and quickly, and then add the controls that the user needs, and finally create a visual graphical application interface. This article introduces the development process of the visual interface in ABAQUS in more detail. First, after starting ABAQUS/CAE, click on [Plug-ins menu] and select [ABAQUS] from the drop-down menu, then click on the [RSG Dialog Builder] in the menu that continues to appear, and the graphical interface creation dialog box shown in Figure 4 will pop up. In the dialog box, you can create a visual interface and add and modify titles, content, appearance, and functionality. In the process of setting up the development interface, you can click the [Show Dialog] button to pop up the dialog box for the graphic interactive interface that is being edited, which is to display the dialog box that has already been constructed. The establishment of graphical visual interactive interface is mainly accomplished through the following steps:

(1) Change [Title] to [Pipeline Steel Parametric Modeling Program].

(2) Click the [Group Box] button in the left part of the second part of the toolbar and add a group box under the title. The purpose of adding a group box is to facilitate the interface division and make the interface more intuitive.

(3) Click the [Text Filed] button in the fourth part of the left side of the toolbar to add a text edit box. The text label is the content corresponding to the input parameters. The default input content can be set to [Part Name], and you can click [Show Dialog] to view the interface after setting.
(4) Edit the interface in the same way and insert images.

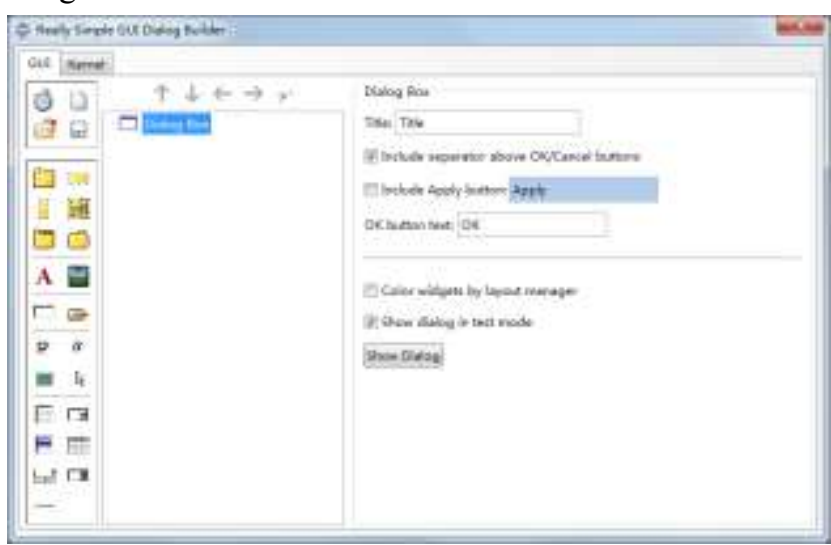

Figure 4. RSG (Really Simple GUI) Dialog Builder.

After the establishment of the user graphic interactive interface is completed, the corresponding Python script file needs to be selected as a module name on the Kernel tab page. Select the script file written in Python, and then select the corresponding function expression relationship, and connect the dialog and ABAQUS kernel script through the "module. Name. function. Name" command. After the interface is developed, you can directly save the plug-in program. After the interface is developed, you can directly save the plug-in program. All relevant file information contained in the plug-in program is stored in the ABAQUS plug-in directory.

\subsection{The development of kernel script}

This article uses a relatively quick way to develop a kernel script. When performing various operations in ABAQUS/CAE, the background program will be recorded in the "Abaqus.rpy" file in the current working directory in real time. In addition, when each time the CAE model is saved, a .jnl file with the same name as the model name is automatically saved in the directory where the file is saved. The script commands used in the entire modeling process are recorded in files whose suffixes are ".rpy" and ".jnl". The above two types of files are opened by using text editing software, the contents of the script records are analyzed, and the most optimized instructions are extracted according to their own requirements. Then modify the instructions, edit the corresponding function with the visual interface, you can quickly edit the kernel script. In this way, we can save a lot of script editing time and improve the efficiency of scripting by users. 


\section{A case study of interactive interface}

First open the plug-in program in ABAQUS, as shown in Figure 5. The size of the sheet material chosen in this paper is width $\mathrm{W}=1219 \mathrm{~mm}$, thickness $\mathrm{T}=33 \mathrm{~mm}$, and length $\mathrm{L}=600 \mathrm{~mm}$. The material of the sheet is $\mathrm{X} 80$ pipeline steel with a yield strength of $445 \mathrm{Mpa}$. First input the corresponding parameters into the dialog box, and then click the OK button to begin the establishment of the finite element model. Figure 6 is a screenshot in the automatic modeling process. The automatic modeling essentially establishes operation commands through plug-ins, and then quickly builds the model.

It takes only about 2 minutes to build the model and build all the components. Figure 7 and Figure 8 show the assembled model. The workload of building models in the interactive interface is greatly simplified compared to that in ABAQUS, and at the same time it provides convenience for researchers in practical projects.

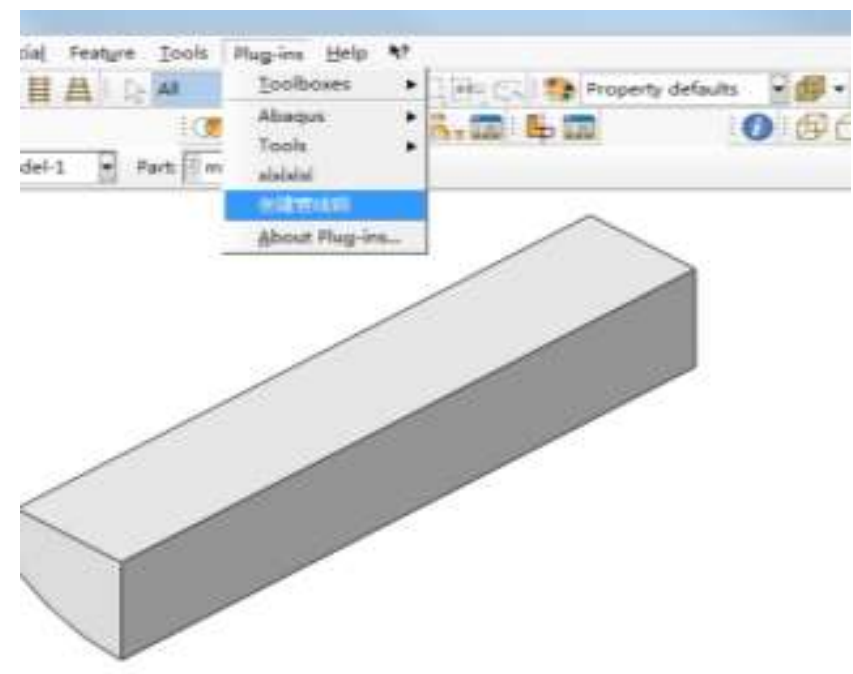

Figure 5. Open the ABAQUS plug-in.

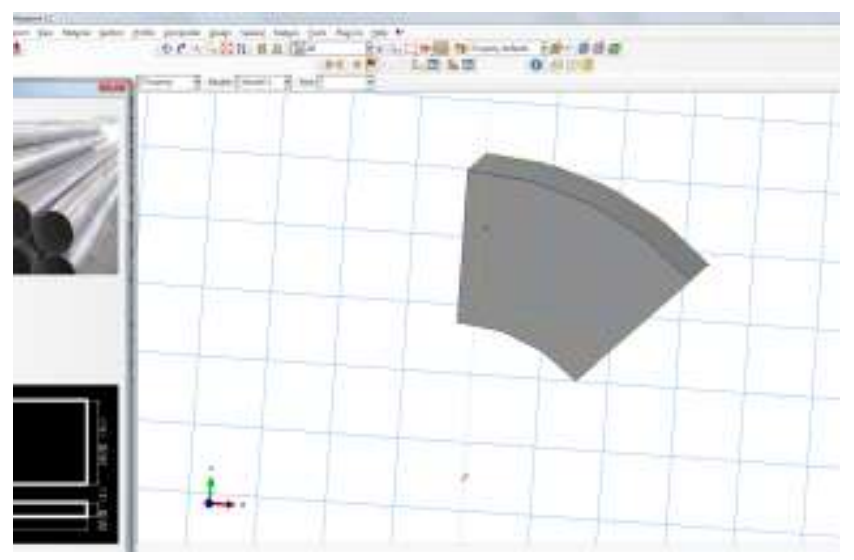

Figure 6. Automated modeling process.

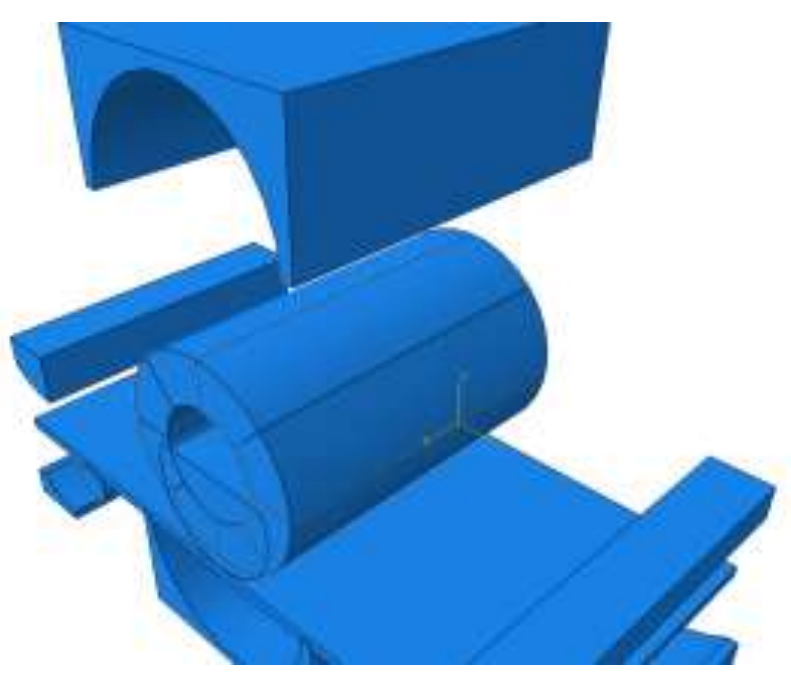

Figure 7. 3D view after modeling.
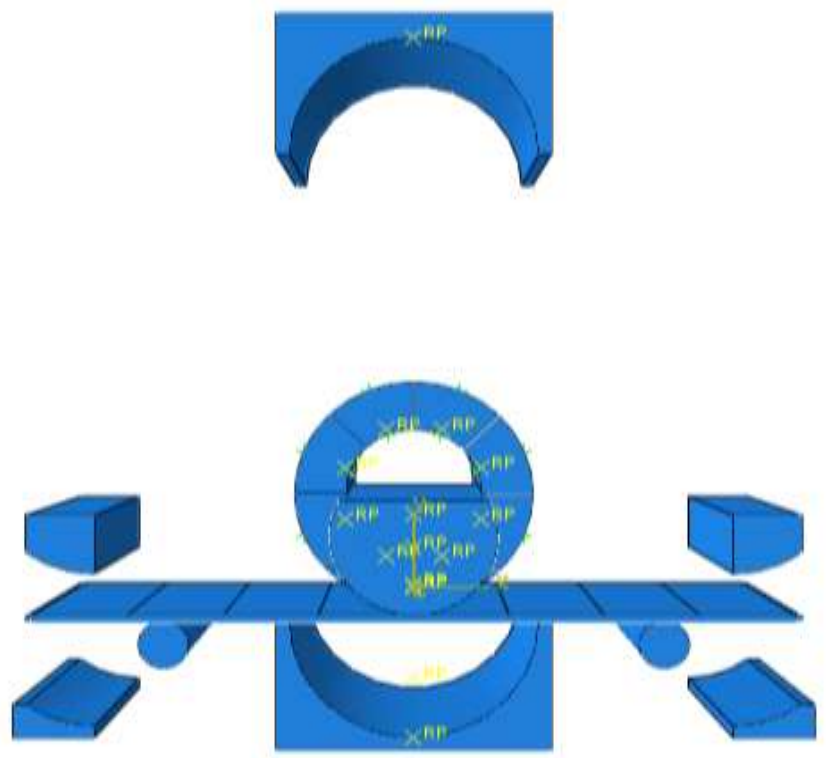

Figure 8. Front view after modeling is complete.

\section{Conclusion}

In this paper, a visual graphic interface is established by writing a Python language script, and parametric modeling of the sheet and mold in the UOE molding process is realized. For the problems of complex model and difficult modeling, the modeling method of visual graphical interface can be used to solve it, and the model can be established quickly and automatically. Whether in scientific research or in actual engineering, the developed visual interface can effectively reduce working hours, improve work efficiency, and provide assistance for engineers and scholars.

\section{References}


[1] J. Teng, H. Zhang, Z.H. Li. Journal of Disaster

Prevention and Mitigation Engineering, 33,9 (2013).

[2] R.M. Xie, X.J. Wang. China Water Transport, 17, 77 (2017).

[3] X.D. Zhang, J. Liu, S.G. Zou, W.Y. Tang. Journal of Plasticity Engineering, 24, 140 (2017).
[4] H. Wang, T.C. Li, Q.B. Wang, Y.B. Zhan. Modern Tunnelling Technology, 52, 160 (2015).

[5] Q. Zhang, Y. Ma, S.C. Li. Ship Electronic Engineering, 31, 131 (2011).

[6] H.W. Li, W.D. Wang. Journal of Building Structures, 34, 353 (2013). 